\title{
Expression analysis of $\alpha$-smooth muscle actin and tenascin- $C$ in the periodontal ligament under orthodontic loading or in vitro culture
}

\author{
Hui Xu ${ }^{1}$, Ding Bai ${ }^{1}$, L-Bruno Ruest ${ }^{2}$, Jian Q Feng ${ }^{2}$, Yong-Wen Guo ${ }^{1}$, Ye Tian ${ }^{1}$, Yan Jing ${ }^{1}$, Yao He ${ }^{1}$ \\ and Xiang-Long Han $^{1,2}$
}

$\alpha$-smooth muscle actin ( $\alpha$-SMA) and tenascin-C are stress-induced phenotypic features of myofibroblasts. The expression levels of these two proteins closely correlate with the extracellular mechanical microenvironment. We investigated how the expression of $\alpha$-SMA and tenascin- $\mathrm{C}$ was altered in the periodontal ligament (PDL) under orthodontic loading to indirectly reveal the intrinsic mechanical microenvironment in the PDL. In this study, we demonstrated the synergistic effects of transforming growth factor- $\beta 1$ (TGF- $\beta 1$ ) and mechanical tensile or compressive stress on myofibroblast differentiation from human periodontal ligament cells (hPDLCs). The hPDLCs under higher tensile or compressive stress significantly increased their levels of $\alpha$-SMA and tenascin-C compared with those under lower tensile or compressive stress. A similar trend was observed in the tension and compression areas of the PDL under continuous light or heavy orthodontic load in rats. During the time-course analysis of expression, we observed that an increase in $\alpha$-SMA levels was matched by an increase in tenascin-C levels in the PDL under orthodontic load in vivo. The time-dependent variation of $\alpha$-SMA and tenascin-C expression in the PDL may indicate the time-dependent variation of intrinsic stress under constant extrinsic loading.

International Journal of Oral Science (2015) 7, 232-241; doi:10.1038/ijos.2015.26; published 11 September 2015

Keywords: $\alpha$-smooth muscle actin; mechanical load; myofibroblast; periodontal ligament; tenascin-C; transforming growth factor- $\beta 1$

\section{INTRODUCTION}

Orthodontic mechanotherapy is aimed at tooth movement by remodeling and adaptive changes in paradental tissues. To achieve this effect, the extrinsic orthodontic load on the tooth is transferred to an intrinsic mechanical stimulus that evokes cellular responses that interfere with the physiological equilibrium of the tooth-alveolus complex. ${ }^{1}$ In fact, the intrinsic stresses or strains within the paradental tissues, not the extrinsic load, are sensed by the cells and control the remodeling process. ${ }^{2}$

Attempts have long been made to obtain insight into the intrinsic mechanical microenvironment in the periodontal ligament (PDL). With the available instrumentation, measuring precisely the amount of stress exerted on the PDL cells or on the alveolar bones under orthodontic load is impossible. Biomechanical studies have suggested that the force applied to the tooth differs from the resulting stresses or strains within the PDL in both magnitude and time-dependent variation. ${ }^{2-4}$ However, these studies addressed the biomechanical issues using mathematic models to analyze the viscoelastic properties of the PDL. ${ }^{2-6}$ The mathematic data, which were based on tooth-PDL-bone segment specimens mounted in testing machines, could not completely represent the actual conditions in living tissue.

The discovery of myofibroblasts in the PDL has provided new perspectives for understanding the biomechanical mechanisms involved in orthodontic tooth movement. ${ }^{7}$ Myofibroblasts are mechanically active and contribute to alterations in the extracellular mechanical microenvironment and to overall tissue mechanics. ${ }^{8-9}$ The expression of $\alpha$-smooth muscle actin ( $\alpha$-SMA) defines the phenotypic transition to myofibroblasts and is a primary contributor to enhanced contractile force generation by these cells. ${ }^{9}$ Myofibroblasts could be identified more reliably and specifically by the co-expression of vimentin and $\alpha$-SMA without the expression of desmin..$^{10-11}$ Moreover, myofibroblasts also express tenascin- $\mathrm{C}$, which is a prominent extracellular matrix (ECM) protein regulated by mechanical stress. ${ }^{10-12}$ The secretion of tenascin-C helps cells to loosen their matrix adhesion contacts to avoid overstretching in tissues under high mechanical load, functioning as a "shock absorber" for mechanical stress. ${ }^{12-14}$

A greater understanding of this process emerges when the stressinduced myofibroblastic phenotype and the extracellular mechanical microenvironment are linked. Cells are generally acknowledged to live

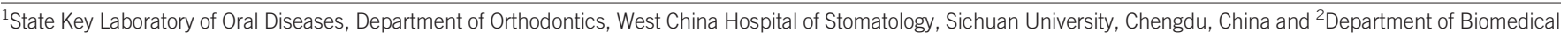
Sciences, Baylor College of Dentistry, Texas A\&M University, Dallas, USA

Correspondence: Dr. XL Han, Department of Biomedical Sciences, Baylor College of Dentistry, Texas A\&M University, 3302 Gaston Ave, Dallas TX 75246, USA

E-mail: xhan@scu.edu.cn

Accepted 22 May 2015
} 
in a "mechanical niche" that provides a set of mechanical cues that are crucial for maintaining both cellular phenotype and identity. ${ }^{8,15-17}$ In turn, mechanically inducible alterations of cellular phenotypes indicate an altered mechanical microenvironment. The expression of $\alpha$-SMA and tenascin- $C$ is a stress-induced phenotypic feature of myofibroblasts in vivo and in vitro. ${ }^{12-14,18}$ This feature not only enables the myofibroblast to adapt its activity to changes in the mechanical microenvironment but also to adjust its contractile force generation to alter the extracellular stress in the ECM. ${ }^{8,18-19}$ Thus, the expression of $\alpha$-SMA and tenascin-C might provide insight regarding the intrinsic mechanical microenvironment in the PDL.

However, three issues need to be addressed before we can infer the in vivo mechanical microenvironment from $\alpha$-SMA and tenascin- $\mathrm{C}$ expression levels in the PDL under orthodontic load. First, in vitro experiments need to be performed to confirm that the PDL cells undergo altered expression levels of $\alpha$-SMA and tenascin- $C$ in response to mechanical load exerted directly on the cells and to investigate the relationship between protein expression levels and load pattern and magnitude. Second, the biochemical stimuli regulating these protein expression levels need to be defined. Transforming growth factor- $\beta$ (TGF- $\beta$ ) is known to play a role in myofibroblast differentiation during tissue fibrosis. ${ }^{18,20}$ Third, the comparison between the in vivo and in vitro induction conditions should be optimized in design but objectively evaluated. The above issues have not yet been clarified.

The objectives of this study were to analyze the expression of $\alpha$-SMA and tenascin- $\mathrm{C}$ over time in the PDL under in vivo orthodontic load and in vitro mechanical stress and to investigate the effect of TGF- $\beta 1$ on stress-induced expression of $\alpha$-SMA and tenascin-C in hPDLCs.

\section{MATERIALS AND METHODS}

\section{Animals}

Animal interventions were approved by the Institutional Research Ethics Committee. Eighty-four 8-week-old $(200 \pm 20)$ g male SpragueDawley rats were used and divided into two groups, the light-force group and the heavy-force group. The rats were maintained in a temperature-controlled room $\left(25-28{ }^{\circ} \mathrm{C}\right)$ with a 12/12-hour light-dark cycle. We used a split-mouth model for the experiments. After each rat was anesthetized by an intraperitoneal injection of chloral hydrate $\left(3 \mathrm{~mL} \cdot \mathrm{kg}^{-1}\right)$, an orthodontic appliance consisting of a $6-\mathrm{mm}$ length of closed-coil spring (Unitek 3M, Monrovia, CA, USA) was fixed to exert continuous force to the maxillary first molar, with the maxillary incisor serving as anchorage. The teeth on the right side were ligated to the appliance with the spring activated to exert an initial force of $20 \mathrm{~g}$ for the light-force group and $60 \mathrm{~g}$ for the heavy-force group, with no reactivation during the experiment. The teeth on the left side served as controls, with the spring ligated to the teeth but maintained in an inactive state. For each group, the 42 rats were divided into seven subgroups. The teeth from subgroups 1-7 were subjected to orthodontic load or to unloaded control for 1, 3, 5, 7, 14, 21, and 28 days, respectively.

Rats were euthanized with an overdose of anesthesia at the indicated time points, and the maxillas were removed and fixed. The distance between the first and second maxillary molars was measured using a vernier caliper with accuracy of $0.02 \mathrm{~mm}$. All measurements were performed twice by two investigators independently within a few minutes. Interexaminer reliability was assessed using the intraclass coefficient of correlation (ICC). The maxillas were scanned by a micro-computed tomography (CT) scanner. The demineralized maxillary tissues were dehydrated, embedded in paraffin, and then cut into $5-\mu \mathrm{m}$ thick antero-posterior serial sections for immunohistochemical study.

\section{Cell culture}

Human periodontal ligament cells (hPDLCs) were prepared following the method described by Palmon et al. ${ }^{21}$ hPDLCs were isolated from the healthy PDL of premolar teeth of adults undergoing tooth extraction for orthodontic reasons. All patients gave informed consent before providing the samples. The periodontal tissues were obtained from the central third of the roots. The cells were cultured in Dulbecco's modified Eagle medium (DMEM) containing 10\% fetal bovine serum (FBS) for 7 days at $37{ }^{\circ} \mathrm{C}$ under $5 \% \mathrm{CO}_{2}$ and then passaged at a 1:3 split ratio after reaching confluence. The cells between passages 3 and 5 were used in the experiments.

\section{Mechanical stress application and drug administration}

The hPDLCs were seeded onto $15 \mathrm{~cm}^{2}(5 \mathrm{~cm} \times 3 \mathrm{~cm})$ cell culture plates at a density of $5 \times 10^{4}$ cells per $\mathrm{cm}^{2}$ and cultured in DMEM plus $10 \%$ FBS. After reaching $80 \%$ confluence, cell growth was arrested by incubating the hPDLCs with serum-free medium for 24 hours. Afterwards, the quiescent cells were maintained in DMEM with $1 \%$ FBS. For vehicle control, the cells were maintained in DMEM plus $1 \%$ FBS. For drug administration, recombinant human TGF- $\beta 1$ ( $5 \mathrm{ng} \cdot \mathrm{mL}^{-1}$; Protech Technology, Sparks, NV, USA) was added to the culture medium. For stress application, the cells were exposed to cyclic uniaxial tension or compression (magnitude of 2000 or $4000 \mu$ strain, frequency of $0.5 \mathrm{~Hz}$ ) supplied by a uniaxial four-point bending system (developed at Sichuan University, patents CN2534576 and $\mathrm{CN} 1425905)$ in the incubator. Experimental procedures were repeated three times for each experimental condition.

For the hPDLCs subjected to mechanical loading (2 000 or $4000 \mu$ strain) for $0,1,3,6$, or 12 hours, cultures were harvested at each time point, $\alpha$-SMA expression was analyzed by real-time polymerase chain reaction (RT-PCR) and immunocytochemistry, and TGF- $\beta 1$ production in the supernatant was evaluated by enzyme-linked immunosorbent assay (ELISA). For hPDLCs subjected to $4000 \mu$ strain of mechanical loading for 12 hours, cells were harvested, and flow cytometry was performed. For the TGF- $\beta 1$-treated hPDLCs (with simultaneous stress application or in static cultures for 6 hours), the expression levels of $\alpha$-SMA and tenascin- $C$ were evaluated by RT-PCR, immunocytochemistry and immunofluorescence.

\section{Immunochemistry and immunofluorescence}

The primary antibodies used were as follows: mouse monoclonal anti- $\alpha$-SMA (clone: 1 A4; dilution: 1:200), rabbit monoclonal anti-tenascin-C (clone: EPR4219; dilution: 1:200), mouse monoclonal anticytokeratin (clone: M20; dilution: 1:200), rabbit monoclonal antivimentin (clone: EPR3776; dilution: 1:200), and rabbit monoclonal anti-desmin (clone: Y66; dilution: 1:200), all from Abcam (Cambridge, UK). For immunochemical staining, biotinylated horse anti-mouse immunoglobulin $\mathrm{G}$ (IgG) and goat anti-rabbit IgG (dilution: 1:100; Zhongshan Biotechnology, Beijing, China) were used as secondary antibodies. The reaction products were visualized in brown using a diaminobenzidine substrate kit (DAB; Zhongshan Biotechnology, Beijing, China). Counterstaining was performed with haematoxylin or methyl green. For immunofluorescence, Alexa Fluor 488 donkey anti-rabbit IgG and Alexa Fluor 594 donkey anti-mouse IgG (dilution: 1:500; Invitrogen, Carlsbad, CA, USA) were used as secondary antibodies. diamidino-phenyl-indole (DAPI; dilution: 1:500; Invitrogen, Carlsbad, CA, USA) was used to stain the nuclei. Control staining was performed by substituting 
non-immune serum (or phosphate-buffered saline) for the primary antibodies. Fluorescence images were acquired using an inverted fluorescence microscope (IX71; Olympus, Tokyo, Japan).

\section{Image analysis}

While viewed under a microscope (E600; Nikon, Tokyo, Japan), the study areas were manually selected in the PDL anterior or posterior to the cervical third of the roots of the maxillary first molars, at a constant distance from the root surfaces. The sizes of the study areas and the integrated optical density (IOD) of the positive stains were measured using Image-Pro Plus 5.0 (Media Cybernetics, Bethesda, MD, USA). The expression levels of the proteins were measured by the mean optical density (MOD; MOD = IOD per unit of study area).

\section{ELISA}

The TGF- $\beta 1$ levels in the collected supernatant were measured by ELISA (R\&D Systems, Oxon, UK) according to the manufacturer's specifications. Horseradish peroxidase-conjugated secondary antibodies were used to quantify the TGF- $\beta 1$ levels and detected by incubation with tetramethyl benzidine at $37^{\circ} \mathrm{C}$. Then, the enzymatic reaction was stopped, and the absorbance was read at $450 \mathrm{~nm}$.

\section{RT-PCR}

RT-PCR was used to determine the expression of $\alpha$-SMA and tenascin-C mRNA transcripts. Experiments were performed according to the manufacturer's guidelines. Total RNA was extracted from cultured cells using TRIzol reagent (Invitrogen, Carlsbad, CA, USA). Before first-strand cDNA synthesis, the RNA pellet was dried and dissolved in nuclease-free water, and the RNA concentration was measured spectrophotometrically. Only those samples of high quality (A260/ A280 ratios ranging from 1.80 to 1.99 ) were used in the analysis. cDNA was reverse transcribed from $8 \mu \mathrm{L}$ of total cellular RNA using a TaKaRa PrimeScript 1st Strand cDNA Synthesis Kit (TaKaRa, Tokyo, Japan). In total, $20 \mu \mathrm{L}$ of the cDNA mixture was subjected to PCR amplification. The targeted genes were amplified using the following primers: 5'-TGGCTATTCCTTCGTTACTACTGCT-3' (sense primer) and 5'-CATCAGGCAACTCGTAACTCTTCTC-3' (anti-sense primer) for the human $\alpha$-SMA gene, and $5^{\prime}$-TGGAGT ATGCTCTGACCGACCT- $3^{\prime}$ (sense primer) and 5'-GTTGTGAACTT GGCAGTGATGG-3' (anti-sense primer) for the human tenascin-C gene. The amplification of glyceraldehyde-3-phosphate dehydrogenase $(\mathrm{GAPDH})$ was used as an internal control.

\section{Statistics}

Data analysis was performed using SPSS (Statistical Package for the Social Sciences) 13.0 software (SPSS, Chicago, IL, USA). Descriptive statistics were calculated for each parameter. Interexaminer reliability was assessed by ICC. Comparisons were performed by analysis of variance (ANOVA), followed by the LSD and S-N-K methods for multiple comparisons between pairs. A probability at the $5 \%$ level or less $(P<0.05)$ was considered statistically significant. The data are presented as the mean \pm standard deviation of triplicates.

\section{RESULTS}

Cyclic uniaxial tensile or compressive stress synergized with TGF- $\beta 1$ to promote hPDLC differentiation into myofibroblasts The hPDLCs cultured in vitro showed a fibroblast-like phenotype, with positive immunolabeling for vimentin but negative immunolabeling for desmin and cytokeratin. The hPDLCs acquired a myofibroblast phenotype with exogenous TGF- $\beta 1$ stimulation $\left(5 \mathrm{ng} \cdot \mathrm{mL}^{-1}\right)$, characterized by positive immunolabeling for vimentin and $\alpha$-SMA-containing stress fibers but negative immunolabeling for desmin and cytokeratin (Figure 1a). Immunochemical identification was performed, and similar results were observed in cultures exposed to mechanical loading (magnitude of 2000 or $4000 \mu$ strain, tension or compression) alone or to stress plus TGF- $\beta 1$ treatment.

For the hPDLCs subjected to $4000 \mu$ strain of mechanical load for 12 hours, flow cytometry showed an almost unchanged $(P>0.05)$ ratio of cells at $G_{0}$ phase to $G_{1}$ phase, suggesting that the mechanical load did not have a significant effect on cell survival and proliferation.
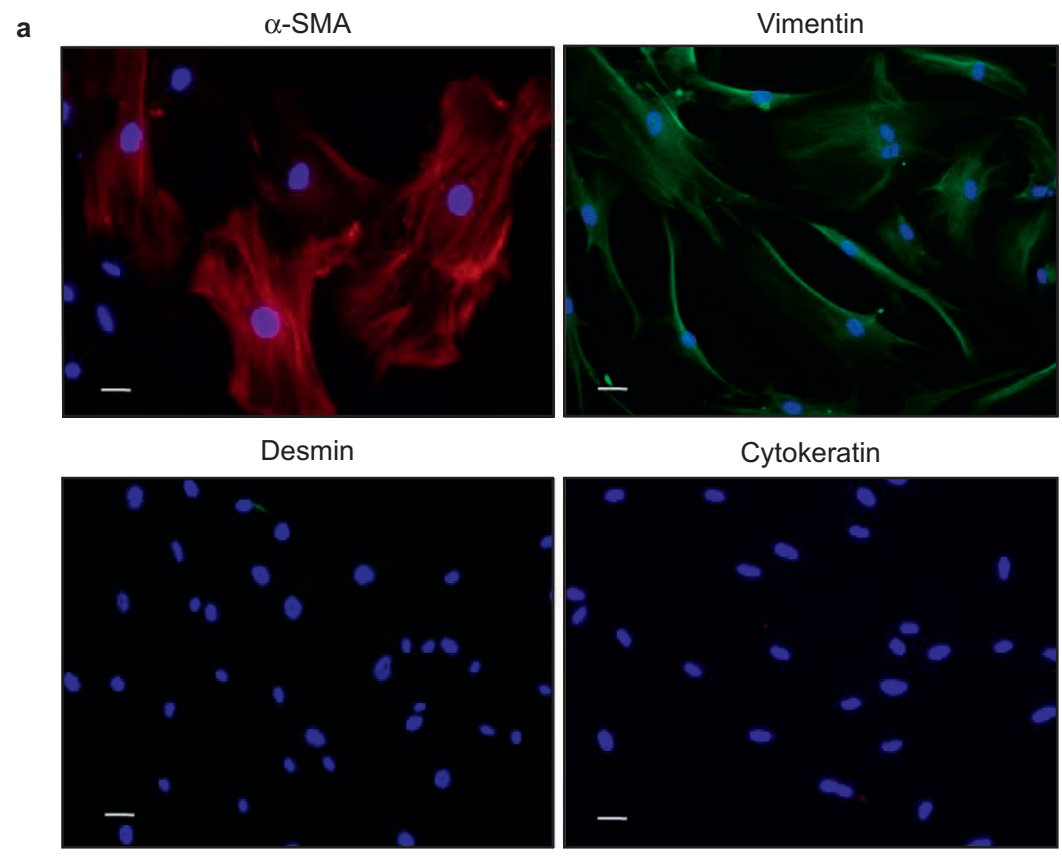
b
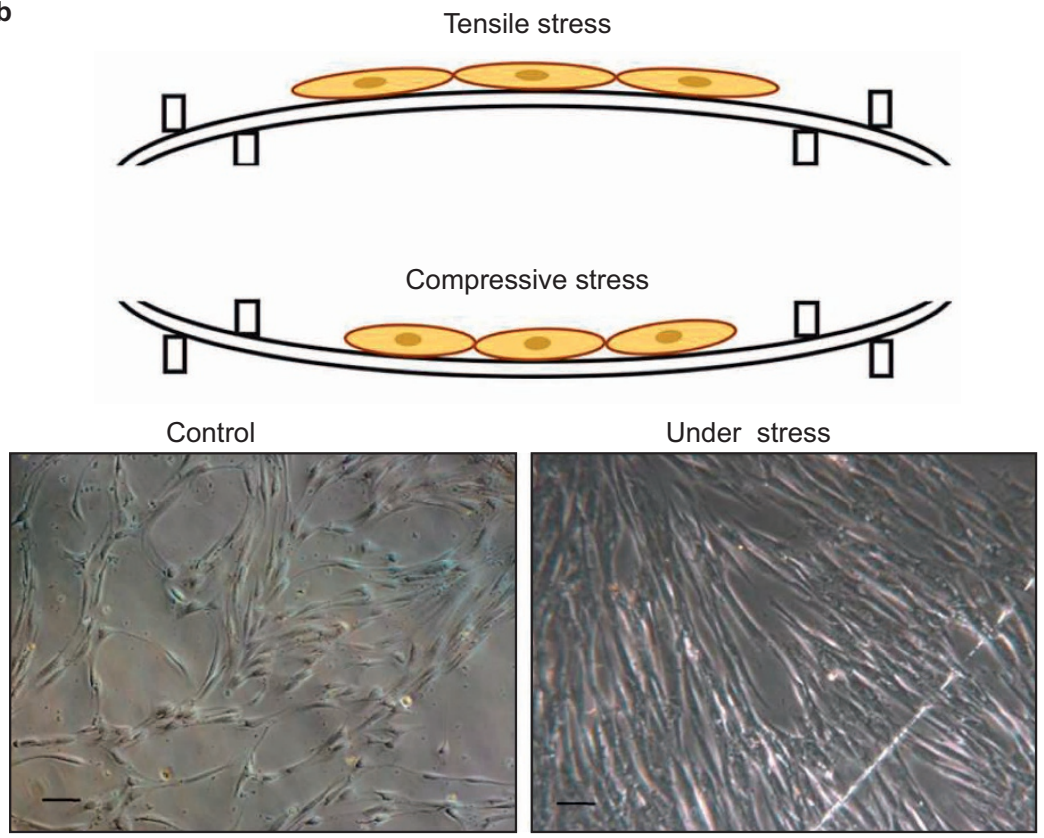

C
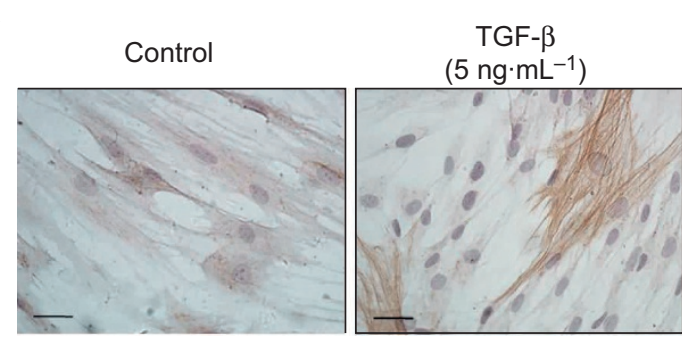

Tension
(2 $000 \mu$ strain)

Tension
(4 $000 \mu$ strain $)$

Tension (4000 $\mu$ strain)
+ TGF- $\beta$
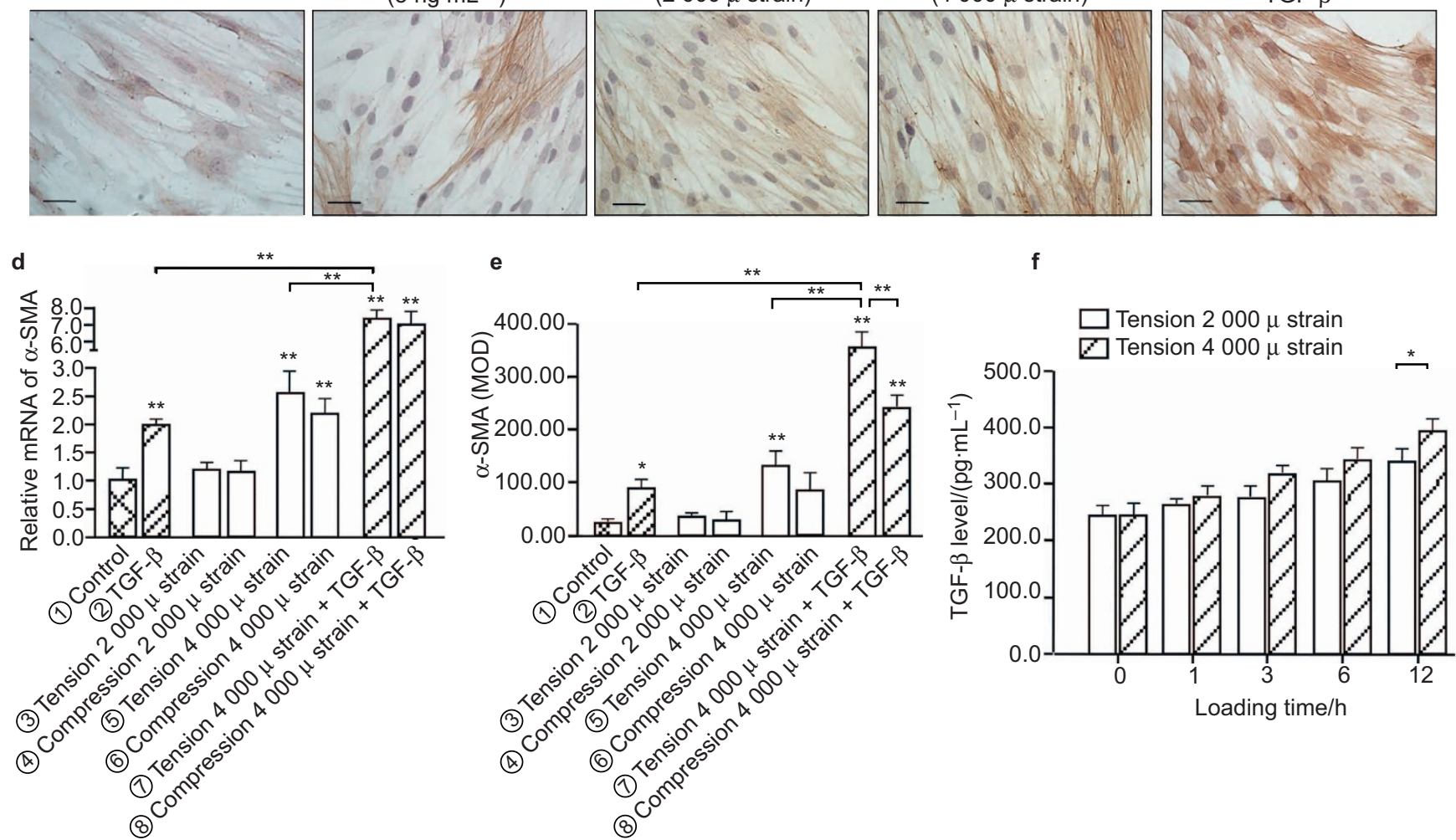

Figure 1 Cyclic uniaxial tensile or compressive stress synergized with TGF- $\beta 1$ to promote the phenotypic transition from hPDLCs to myofibroblasts. (a) Positive immunolabeling for vimentin and $\alpha$-SMA-containing stress fibers but negative immunolabeling for cytokeratin and desmin (scale bar: $20 \mu \mathrm{m}$ )-defined myofibroblast differentiation from hPDLCs after $5 \mathrm{ng} \cdot \mathrm{mL}^{-1}$ TGF- $\beta 1$ treatment. (b) Schematic diagram of the uniaxial four-point bending system. When viewed under a microscope, the static cultured hPDLCs showed a spindle-shaped morphology, whereas the cells under mechanical loading appeared elongated and aligned along the direction of the strain application (scale bar: $50 \mu \mathrm{m}$ ). (c) Representative images of immunochemical staining for $\alpha$-SMA (scale bar: $20 \mu \mathrm{m}$ ) in hPDLCs under various experimental conditions for 6 hours. $\alpha$-SMA expression was evaluated by RT-PCR (d) and semiquantitative immunochemical analysis (e). (f) For hPDLCs exposed to mechanical tension alone for 0 (control), 1, 3, 6, or 12 hours, the concentration of TGF- $\beta 1$ in the culture supernatant was determined at each time point by ELISA. The data are representative of three independent experiments. ${ }^{* *} P<0.01 ; * P<0.05$ vs the control group or between groups as indicated by the line. ELISA, enzyme-linked immunosorbent assay; hPDLC, human periodontal ligament cell; RT-PCR, real time-polymerase chain reaction; SMA, smooth muscle actin; TGF, transforming growth factor. 
a

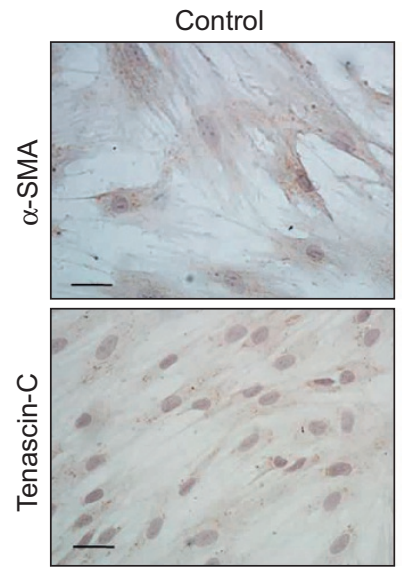

$2000 \mu$ strain + TGF- $\beta$
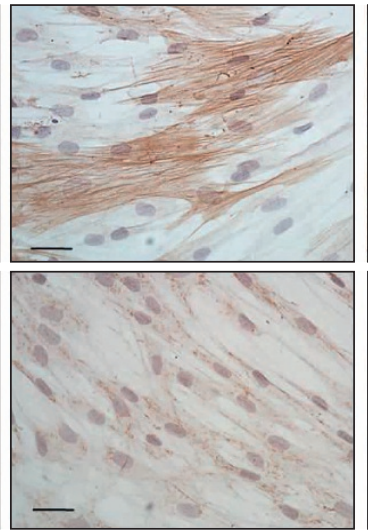

$4000 \mu$ strain + TGF $-\beta$
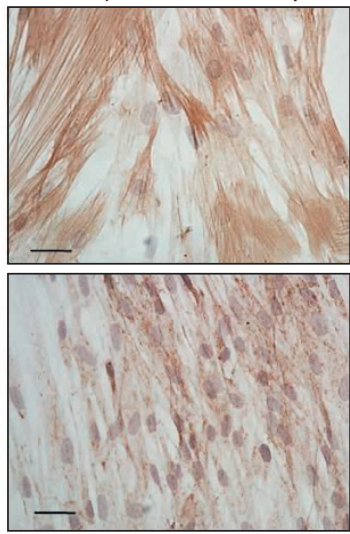

b

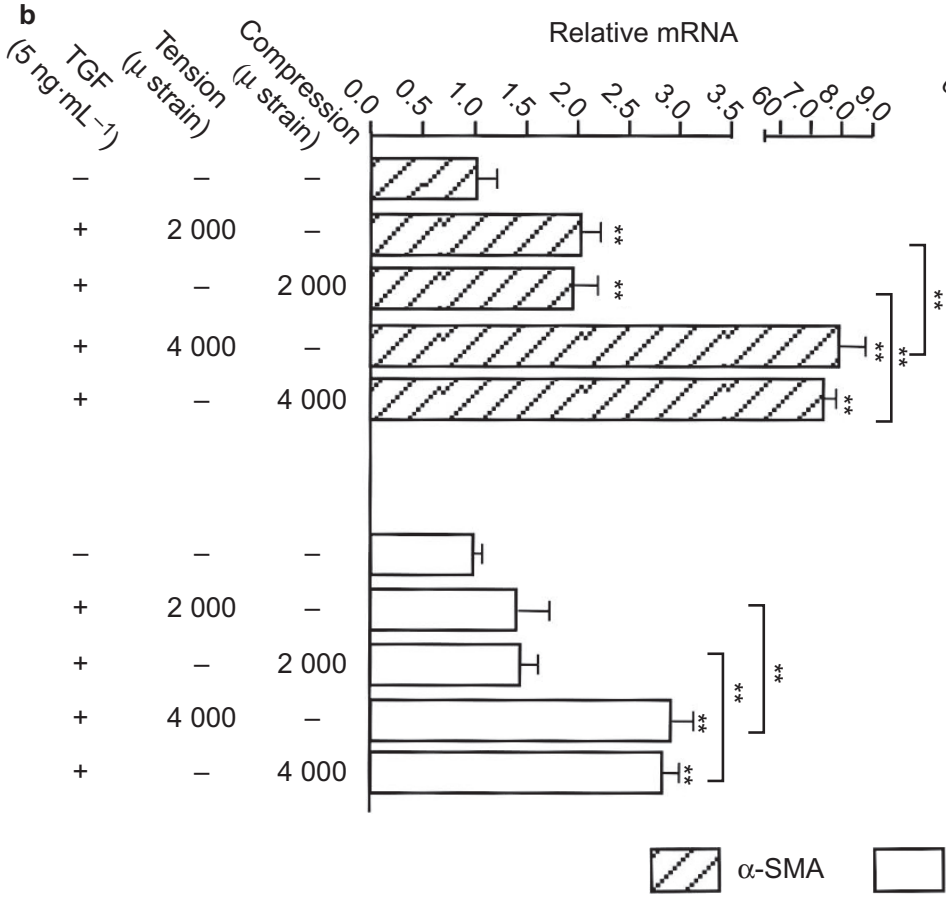

MOD value

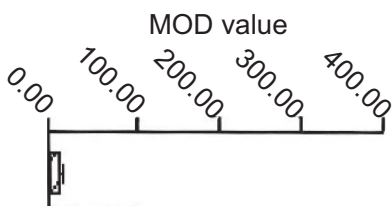

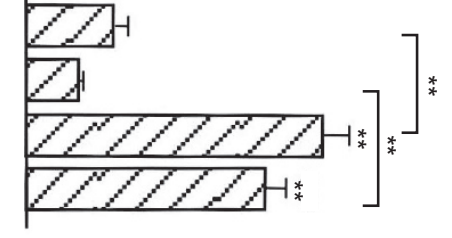
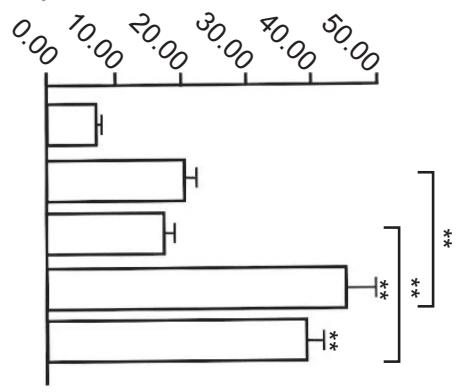

Tenascin-C

Figure 2 Increased expression of $\boldsymbol{\alpha}$-SMA and tenascin-C in response to higher mechanical loads. (a) The expression of $\alpha$-SMA and tenascin-C was immunochemically observed in the hPDLCs (scale bar: $20 \mu \mathrm{m}$ ) after co-stimulation with $5 \mathrm{ng} \cdot \mathrm{mL}^{-1} \mathrm{TGF}-\beta 1$ and different magnitudes of tensile strain. (b) RT-PCR and semiquantitative immunochemical analysis of $\alpha$-SMA and tenascin-C expression levels in the hPDLCs under various experimental conditions. The data are representative of three independent experiments. ${ }^{*} P<0.01$ vs. the control group or between groups as indicated by the line. hPDLC, human periodontal ligament cell; RT-PCR, real time-polymerase chain reaction; SMA, smooth muscle actin; TGF, transforming growth factor.

Myofibroblasts were identified using $\alpha$-SMA as a marker (Figure $1 \mathrm{c}$ and $1 \mathrm{~d}$ ). Weak immunocytochemical reactivity to $\alpha$ SMA was noted in static control cultures and in cultures under 2 $000 \mu$ strain mechanical tension or compression alone. Increased $\alpha$ SMA expression was observed in cultures exposed to $4000 \mu$ strain mechanical loading and in cultures under co-stimulation of $4000 \mu$ strain tension or compression and $5 \mathrm{ng} \cdot \mathrm{mL}^{-1}$ TGF- $\beta 1$ compared with controls $(P<0.01)$. The hPDLCs treated with stress plus TGF- $\beta 1$ showed higher levels of $\alpha$-SMA expression than those under mechanical stimuli or TGF- $\beta 1$ treatment alone (Figure 1c-1e, $P<0.01$ ). Exposing the hPDLCs to $4000 \mu$ strain mechanical tension for 3 hours or more or to $2000 \mu$ strain mechanical tension for 12 hours significantly increased TGF- $\beta 1$ production by the cultured cells compared with static control cultures (Figure 1f, $P<0.01$ ).
A higher magnitude of stress stimulation increased $\alpha$-SMA and tenascin- $\mathrm{C}$ expression compared with a lower magnitude of stress stimulation

In the presence of $5 \mathrm{ng} \cdot \mathrm{mL}^{-1} \mathrm{TGF}-\beta 1$, the hPDLCs under higher mechanical tension or compression displayed significantly increased $\alpha$-SMA $(P<0.01)$ and tenascin-C $(P<0.01)$ expression compared with those under lower tension or compression forces (Figure $2 \mathrm{a}$ and $2 \mathrm{~b}$ ).

Time-dependent variation of $\alpha$-SMA and tenascin-C expression in the rat PDL under continuous orthodontic load

We examined $\alpha$-SMA and tenascin-C expression in tension and compression areas of the PDL under continuous light or heavy orthodontic load (Figures 3-5) immunohistochemically at different time points during 28 days. The unloaded control samples showed negative-to-mild 
$\alpha-S M A$
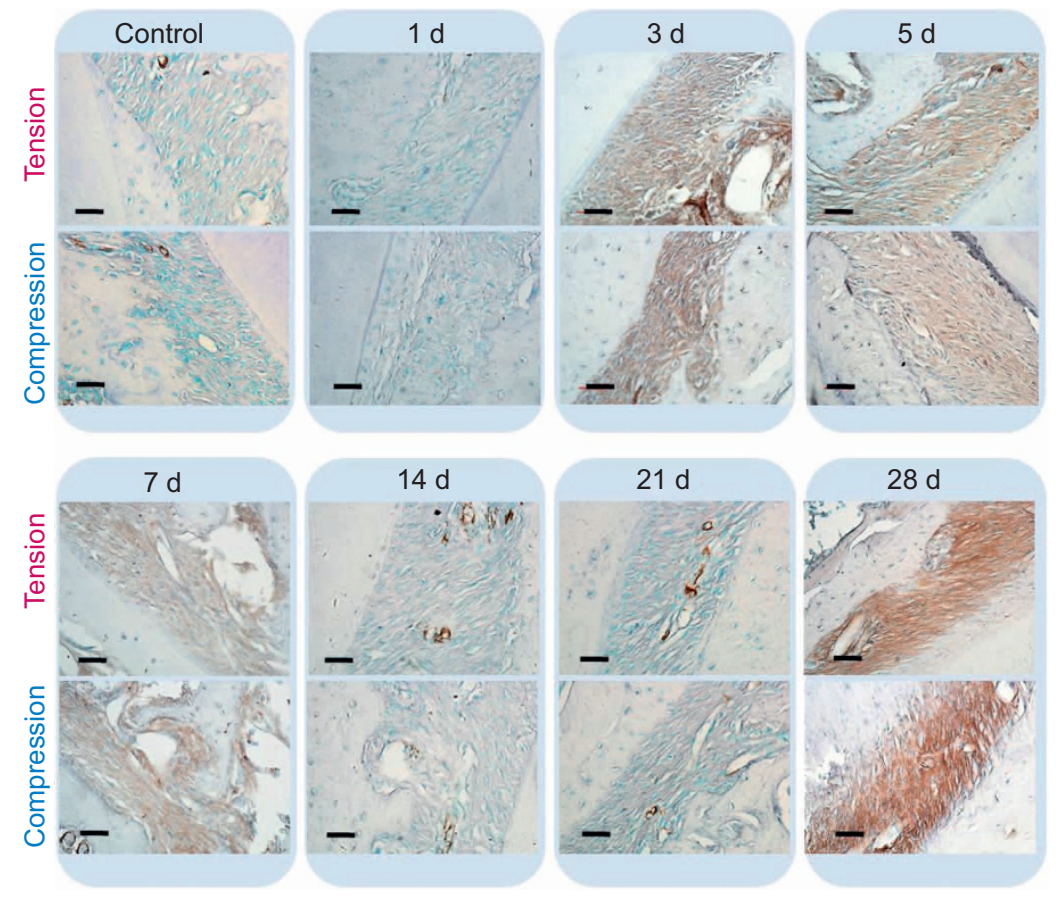

b
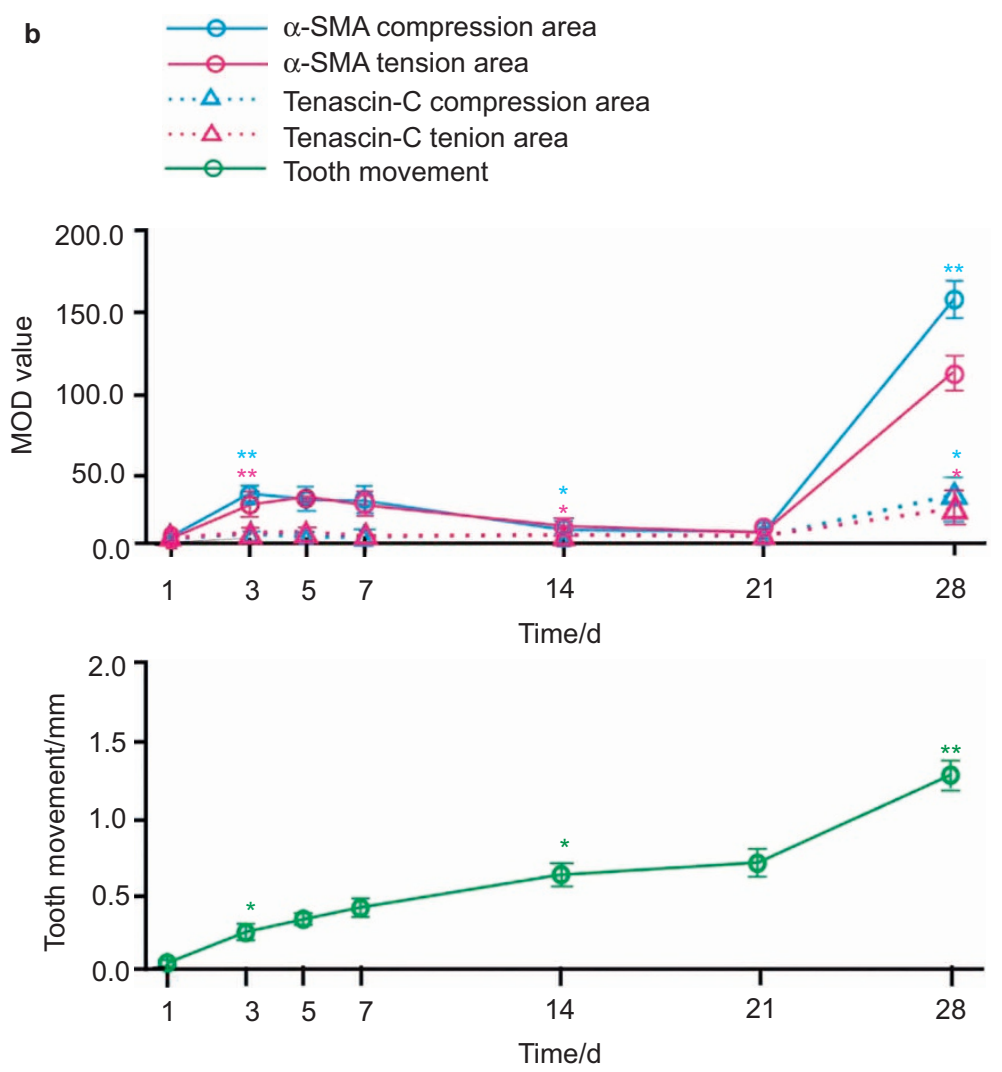

Figure 3 Expression of $\alpha$-SMA and tenascin-C in the tension and compression zones of the PDL under continuous light orthodontic force. (a) Representative immunohistochemical staining results for $\alpha$-SMA under light orthodontic load in the tension and compression zones of the PDL (scale bar: $20 \mu \mathrm{m}$ ). In the light-force panels (a), intense staining for $\alpha$-SMA was observed on the blood vessels in the PDL, while the surrounding tissue showed mild staining. (b) Semiquantitative immunohistochemical analysis of $\alpha$-SMA and tenascin-C expression levels in the tension and compression zones of the PDL under light orthodontic load and the timecourse analysis of distance of tooth movement under light orthodontic force. The data are representative of six independent experiments. $* P<0.05$ vs. the previous time point; ${ }^{*} P<0.01$ vs. the previous time point. PDL, periodontal ligament; SMA, smooth muscle actin. 

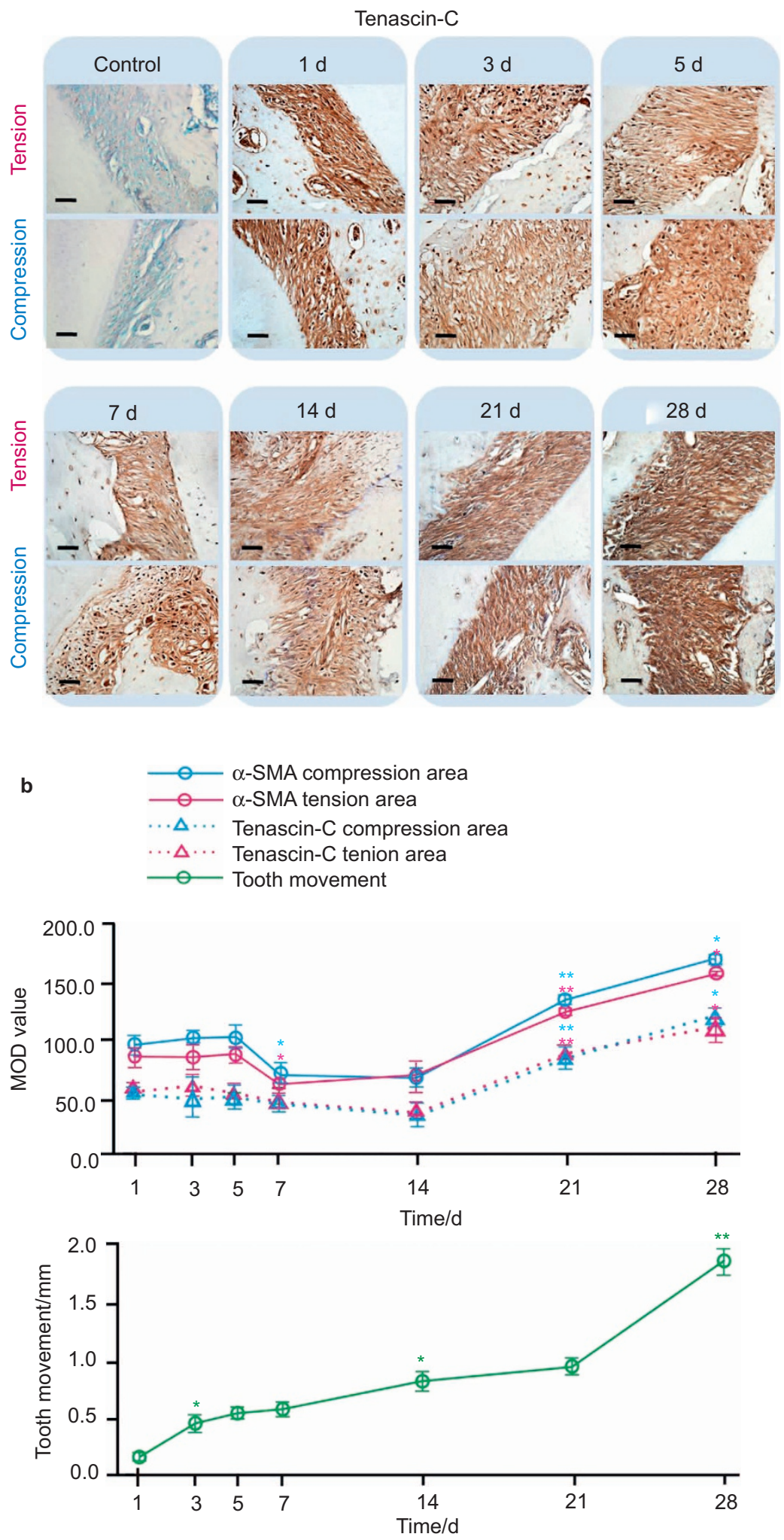

Figure 4 Expression of $\alpha$-SMA and tenascin-C in the tension and compression zones of the PDL under continuous heavy orthodontic force. (a) Representative immunohistochemical staining results for tenascin- $\mathrm{C}$ under heavy orthodontic load in the tension and compression zones of the PDL (scale bar: $20 \mu \mathrm{m}$ ). (b) Semiquantitative immunohistochemical analysis of $\alpha$-SMA and tenascin-C expression levels in the tension and compression zones of the PDL under heavy orthodontic load and the time-course analysis of distance of tooth movement under heavy orthodontic force. The data are representative of six independent experiments. * $P<0.05$ vs. the previous time point; **P<0.01 vs. the previous time point. PDL, periodontal ligament; SMA, smooth muscle actin. 
a

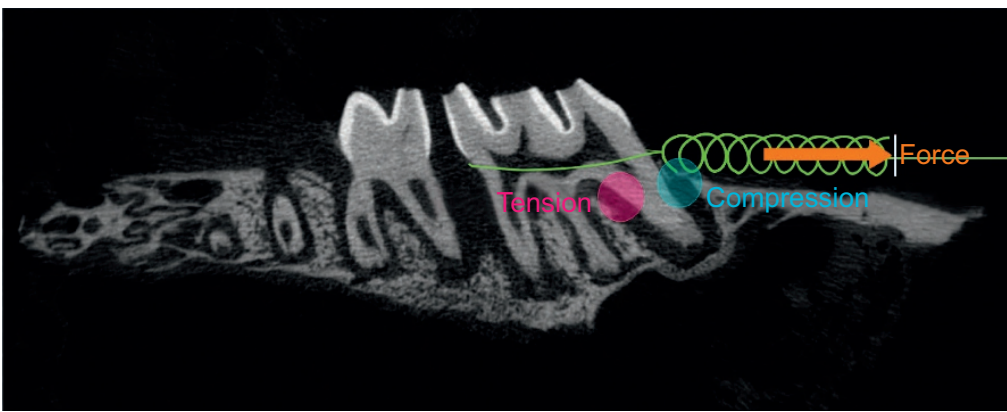

b
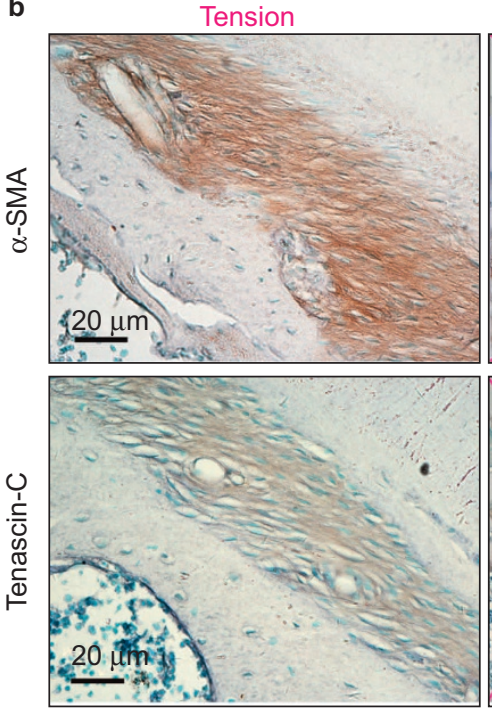
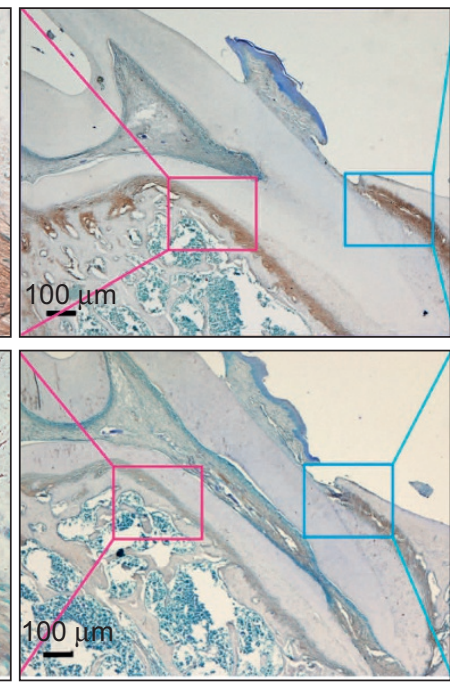

Compression

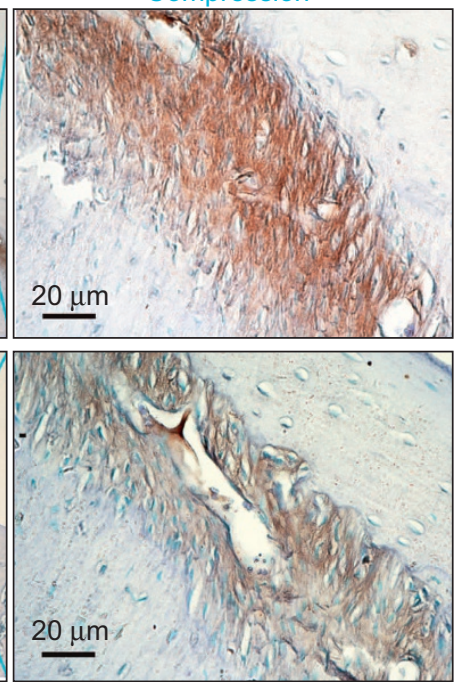

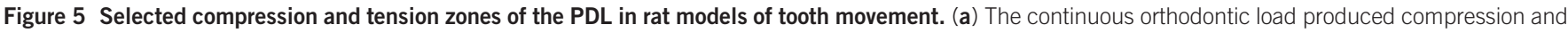

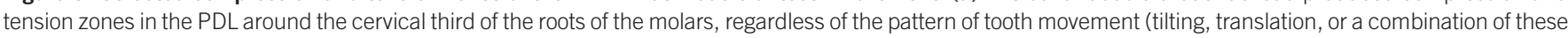

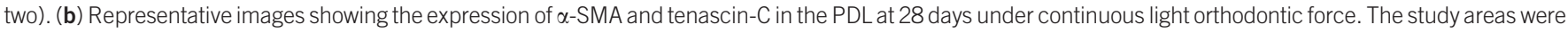
all selected within the compression and tension zones of the PDL. PDL, periodontal ligament; SMA, smooth muscle actin.

staining for $\alpha$-SMA and tenascin-C in the PDL. Under continuous light force (Figure 3), semiquantitative analysis revealed that $\alpha$-SMA levels increased mildly from 1 to 3 days $(P<0.05)$, decreased from 7 to 14 days $(P<0.05)$, and then increased sharply from 21 to 28 days $(P<0.01)$. In contrast, immunolabeling for tenascin- $C$ was negative or weakly positive throughout the observed period, except for an increase from 21 to 28 days $(P<0.05)$. Under continuous heavy force (Figure 4$)$, intense immunocytochemical reactivity to $\alpha$-SMA and tenascin-C was observed initially at 1 day, followed by a slight decrease at 7 days, and then an increase $(P<0.05)$ that was maintained at a relatively high level throughout the duration of observation period. Similar expression trends for $\alpha$-SMA and tenascin-C were found in the PDL on both the tension and compression sides.

In tissues under mechanical load, the expression patterns of tenascin-C and $\alpha$-SMA appeared to be well balanced: negative-to-mild staining for tenascin- $C$ was found in tissues under light load, while the staining became intense under heavy load; likewise, the immunocytochemical reactivity to $\alpha$-SMA increased under higher mechanical load.

\section{The distance of tooth movement under orthodontic load}

The distance of tooth movement was determined by measuring the separation between the first and second maxillary molars. The assessment of interexaminer reliability showed excellent agreement (ICC 0.79-0.92). The mean value of the measurements was used for further statistical analysis. The rate of experimental tooth movement seemed to have three phases (Figures 3-4): a gradual increase from 1 to 14 days $(P<0.05)$, a stationary phase from 14 to 21 days $(P>0.05)$, and a steady increase from 21 to 28 days $(P<0.01)$. The three phases could be divided more distinctly when teeth were under heavy orthodontic load than under light load.

\section{DISCUSSION}

In the present study, the expression of $\alpha$-SMA and tenascin- $C$ increased significantly in the hPDLCs in response to cyclic uniaxial tensile or compressive stress in an in vitro system. The $\alpha$-SMAcontaining stress fibers act as a "cellular contraction enhancer", while tenascin-C-containing stress fibers function as a "shock absorber". ${ }^{9-14}$ A complex mechanism may exist by which PDLCs are capable of determining the balance of extracellular and intracellular stress and of adjusting their contractile activity. Accordingly, tension homeostasis in the PDL is maintained. The expression of $\alpha$-SMA and tenascin-C by the myofibroblasts may play an important role in that mechanism, balancing between "contraction enhancer" and "shock absorber" expression. This balanced expression may explain why an increase in $\alpha$-SMA levels is matched by an increase in tenascin levels and why the expression of these factors increases as the tension load applied to the cells or the tooth intensifies. However, the balance between $\alpha$-SMA and tenascin is slightly skewed in favor of $\alpha$-SMA. 
Our results demonstrated the synergistic effects of TGF- $\beta 1$ and mechanical tensile or compressive stress on the phenotypic conversion of hPDLCs to myofibroblasts. Other factors may also regulate the myofibroblastic phenotype. These factors are likely to be upregulated or downregulated during tooth movement under orthodontic load, which triggers a complex network of biomechanical and biological signaling. ${ }^{1}$ The comparison between the in vivo and in vitro experiments is difficult to define although we investigated the biochemical signaling involved in cell differentiation exhaustively, a limitation of this in vitro study.

However, inferring the in vivo mechanical microenvironment from $\alpha$-SMA and tenascin-C expression in the PDL remains valid for the following three reasons. First, higher expression levels of $\alpha$-SMA and tenascin- $\mathrm{C}$ were detected in cells under a higher magnitude of strain, in the presence or absence of TGF- $\beta 1$ treatment. These results indicate that enhanced mechanical stress promotes myofibroblast differentiation, with or without synergistic biochemical stimuli. Second, Lekic et al. ${ }^{22}$ confirmed the validity of in vitro models for studies of phenotypic regulation in vivo, finding that primary cultured PDL cells are phenotypically similar to the parental cells in the tissues. Notably, the in vivo and in vitro cells should preferentially originate from the same species if comparisons are to be made, as species differences may exist between PDL cells. ${ }^{23-24}$ Third, myofibroblast contractile force and resistant ECM render a positive feedback loop of increased intracellular contractile activity and extracellular stress. ${ }^{8,18-19,25}$ Thus, regardless of how complicated the in vivo signaling network is, inferring that increased $\alpha$-SMA and tenascin-C expression indicates the persistence of myofibroblast differentiation and an increase in intrinsic stress in the PDL is reasonable. The force sensed by the cells in living tissue could not be measured directly; thus, the identification of some indicators that could provide insight regarding the intrinsic mechanical microenvironment would be helpful.

Supported by evidence from previous studies $8,13,18-19,23-24,26-28$ regarding the remodeling of connective tissues and by our observations in this study, we delineated the time-dependent variation of the intrinsic mechanical microenvironment within the PDL under continuous orthodontic load as follows. In the normal PDL tissue, integrins function as links between the ECM and the cytoskeleton. The mechanical resistance of the collagen architecture in the ECM shields embedded cells from mild mechanical load. Orthodontic force leads to microtrauma of the PDL, and the PDL cells are exposed to higher tension (or compression) owing to losing the stress-shielding from the collagen architecture; mechanical loading in conjunction with biochemical stimuli enhances $\alpha$-SMA expression in the PDL cells and induces their differentiation into myofibroblasts. Then, integrin-mediated tissue remodeling of the PDL stimulates the reconstruction of the ECM collagen architecture, which again takes over the mechanical load and thereby shields embedded myofibroblasts from stress, leading to downregulated $\alpha$-SMA expression. Afterwards, continuous orthodontic load produces re-destruction of the collagen architecture and increases force generation within the PDL, causing upregulated $\alpha$-SMA expression; concomitantly, increased tenascin-C expression emerges to protect cells from overstretching. The skewed balance between $\alpha$-SMA and tenascin that we described above may provide enough flexibility to allow tooth movement. This model predicts that larger tooth movement should be accompanied by higher expression levels of both proteins.

From the results of our study, continuous orthodontic force exerted on the tooth may not indicate constant stress within the PDL. The PDL might experience time-dependent variation of intrinsic stress under constant extrinsic loading. The time-dependent expression of $\alpha$-SMA and tenascin- $\mathrm{C}$ indicates intrinsic stress variation in the PDL. Interestingly, we observed three phases of tooth movement: initial speedup (1-3 days), lag (14-21 days), and post-lag acceleration (2128 days), similar to the results previously described. ${ }^{1,28-30}$ Tooth movement tended to accelerate while $\alpha$-SMA expression increased in the PDL and vice versa. The three phases appeared to be divided more distinctly when under a heavier load.

\section{ACKNOWLEDGEMENTS}

This study was funded by National Nature Science Foundation of China (Grant Nos 30970705, 11172190, 81371171, and 81371172).

1 Krishnan V, Davidovitch Z. Cellular, molecular, and tissue-level reactions to orthodontic force. Am J Orthod Dentofacial Orthop 2006; 129(4): 469.e1469.e32.

2 Qian H, Chen J, Katona TR. The influence of PDL principal fibers in a 3-dimensional analysis of orthodontic tooth movement. Am J Orthod Dentofacial Orthop 2001; 120(3): 272-279.

3 Komatsu K, Sanctuary C, Shibata T et al. Stress-relaxation and microscopic dynamics of rabbit periodontal ligament. J Biomech 2007; 40(3): 634-644.

4 Natali AN, Pavan PG, Venturato $C$ et al. Constitutive modeling of the non-linear viscoelasticity of the periodontal ligament. Comput Methods Programs Biomed 2011; 104(2): 193-198.

5 Muraki H, Wakabayashi N, Park I et al. Finite element contact stress analysis of the RPD abutment tooth and periodontal ligament. J Dent 2004; 32(8): 659-665.

6 Zhu YN, Yang WD, Abbott PV et al. The biomechanical role of periodontal ligament in bonded and replanted vertically fractured teeth under cyclic biting forces. Int J Oral Sci 2015; 7(2): 125-130.

7 Meng $Y$, Han X, Huang $L$ et al. Orthodontic mechanical tension effects on the myofibroblast expression of alpha-smooth muscle actin. Angle Orthod 2010; 80(5): 912-918.

8 Hinz B. The myofibroblast: paradigm for a mechanically active cell. J Biomech 2010; 43(1): 146-155.

9 Hinz B, Phan SH, Thannickal VJ et al. Recent developments in myofibroblast biology: paradigms for connective tissue remodeling. Am J Pathol2012; 180(4): 1340-1355.

10 Tomas D, Ulamec M, Hudolin T et al. Myofibroblastic stromal reaction and expression of tenascin-C and laminin in prostate adenocarcinoma. Prostate Cancer Prostatic Dis 2006; 9(4): 414-419.

11 Kaarteenaho-Wiik R, Lakari E, Soini Y et al. Tenascin expression and distribution in pleural inflammatory and fibrotic diseases. J Histochem Cytochem 2000; 48(9): 1257-1268.

12 Chiquet-Ehrismann $\mathrm{R}$, Chiquet $\mathrm{M}$. Tenascins: regulation and putative functions during pathological stress. J Pathol 2003; 200(4): 488-499.

13 Chiquet M, Gelman L, Lutz R et al. From mechanotransduction to extracellular matrix gene expression in fibroblasts. Biochim Biophys Acta 2009; 1793(5): 911-920.

14 Järvinen TA, Józsa $L$, Kannus $P$ et al. Mechanical loading regulates the expression of tenascin- $C$ in the myotendinous junction and tendon but does not induce de novo synthesis in the skeletal muscle. J Cell Sci 2003; 116(Pt 5): 857-866.

15 Discher DE, Janmey P, Wang YL. Tissue cells feel and respond to the stiffness of their substrate. Science 2005; 310(5751): 1139-1143.

16 Janmey PA, McCulloch CA. Cell mechanics: integrating cell responses to mechanical stimuli. Annu Rev Biomed Eng 2007; 9: 1-34.

17 Qi MC, Zou SJ, Han LC et al. Expression of bone-related genes in bone marrow MSCs after cyclic mechanical strain: implications for distraction osteogenesis. Int J Oral Sci 2009; 1(3): 143-150.

18 Tomasek JJ, Gabbiani G, Hinz B et al. Myofibroblasts and mechano-regulation of connective tissue remodelling. Nat Rev Mol Cell Biol 2002; 3(5): 349-363.

19 Wipff PJ, Hinz B. Myofibroblasts work best under stress. J Bodyw Mov Ther 2009; 13(2): 121-127.

20 Wipff PJ, Rifkin DB, Meister JJ et al. Myofibroblast contraction activates latent TGF$\beta 1$ from the extracellular matrix. J Cell Biol 2007; 179(6): 1311-1323.

21 Palmon A, Roos H, Reichenberg $\mathrm{E}$ et al. Basic fibroblast growth factor suppresses tropoelastin gene expression in cultured human periodontal fibroblasts. J Periodont Res 2001; 36(2): 65-70.

22 Lekic $\mathrm{P}$, Rojas J, Birek $\mathrm{C}$ et al. Phenotypic comparison of periodontal ligament cells in vivo and in vitro. J Periodont Res 2001; 36(2): 71-79.

23 Barczyk M, Bolstad AI, Gullberg D. Role of integrins in the periodontal ligament: organizers and facilitators. Periodontol 2000 2013; 63(1): 29-47.

24 Larjava H, Koivisto L, Heino J et al. Integrins in periodontal disease. Exp Cell Res 2014; 325(2): 104-110.

25 Hinz B. Tissue stiffness, latent TGF- $\beta 1$ activation, and mechanical signal transduction: implications for the pathogenesis and treatment of fibrosis. Curr Rheumatol Rep 2009; 11(2): 120-126. 
26 Roy SG, Nozaki Y, Phan SH. Regulation of $\alpha$-smooth muscle actin gene expression in myofibroblast differentiation from rat lung fibroblasts. Int J Biochem Cell Biol 2001 33(7): 723-734.

27 Hinz B, Gabbiani G. Mechanisms of force generation and transmission by myofibroblasts. Curr Opin Biotechnol 2003; 14(5): 538-546.

28 Grinnell F. Fibroblast biology in three-dimensional collagen matrices. Trends Cell Biol 2003; 13(5): 264-269.

29 Sprogar S, Meh A, Vaupotic T et al. Expression levels of endothelin-1, endothelin-2, and endothelin-3 vary during the initial, lag, and late phase of orthodontic tooth movement in rats. Eur J Orthod 2010; 32(3): 324-328.
$30 \mathrm{LiF}, \mathrm{Li} \mathrm{G}, \mathrm{Hu} \mathrm{H}$ et al. Effect of parathyroid hormone on experimental tooth movement in rats. Am J Orthod Dentofacial Orthop 2013; 144(4): 523-532.

(c) (i) $\Theta$ This work is licensed under a Creative Commons Attribution-NonCommercialc. ${ }_{\mathrm{BY}} \mathrm{NC}$ ND NoDerivs 4.0 Unported License. The images or other third party material in this article are included in the article's Creative Commons license, unless indicated otherwise in the credit line; if the material is not included under the Creative Commons license, users will need to obtain permission from the license holder to reproduce the material. To view a copy of this license, visit http://creativecommons.org/licenses/by-nc-nd/4.0/ 